\title{
Psychological and Physiological Changes in Cognitive Functioning in Thalamic Lesion throughout the Rehabilitation Process
}

\author{
Katarzyna Kucharska1, Ewelina Wilkos ${ }^{1}$, Roman Stefański', Grzegorz Makowicz', Danuta Ryglewicz', Ksenia Slawinska ${ }^{2}$, Ewa Piatkowska-
} Janko ${ }^{2}$

${ }^{1}$ Institute of Psychiatry and Neurology, 9 Sobieski 02-957 Warsaw, Poland

${ }^{2}$ Institute of Radioelectronics and Multimedia Technology, Warsaw University of Technology, 15/19 Nowowiejska, 00-665 Warsaw, Poland

\begin{abstract}
Aim: The aim of the study was to assess psychological and physiological changes in cognitive functioning of a thalamic-damaged patient who underwent a stroke rehabilitation program which involved treatment as usual (TAU) combined with a computer-based Neurocognitive Training (NT). Additionally, the healthy control $(\mathrm{HC})$ group $(\mathrm{n}=13)$ was recruited to assess neural circuitry including thalamic involvement in comparison to a patient in acute thalamic stroke who underwent the same scanning procedure before implementation of NT.

Methods: The patient (initials B.D.) was examined at three weeks after the ischemic stroke localized in left tuberothalamic territory. HCs and B.D. were evaluated using various cognitive measures and clinical scales. The computer-based NT was delivered individually once weekly for 60 minutes in a 12-week program. B.D. twice underwent a fMRI scanning session using a block-designed Stroop task: 1) after three weeks from lesion onset, and 2) after therapy.

Results: Compared with HCs, B.D. before therapy demonstrated marked deficits in learning and remembering both verbal and visual material, long-term information storing, problem solving, attentional switching and working memory. After therapy, B.D. showed clinically significant improvement in memory, attention, and executive functions. B.D. showed a post-therapy decreased activation pattern and, by contrasting the congruent versus the incongruent condition, after treatment increased activations remained only in the left parietal lobule and right middle frontal gyrus. In HCs increased activation was detected in right anterior cingulate, frontal regions and striatal region during Stroop interference, which remains in line with other studies.
\end{abstract}

Conclusions: Marked improvement was described in the patient after therapy. Further research is needed to increase knowledge about the involvement of the thalamus in key cognitive processes in the context of efficacy and effectiveness of neurocognitive training.

Keywords: Cognitive deficits; Thalamic stroke; Fmri; Rehabilitation; Neurocognitive training; Stroop task

\section{Introduction}

The involvement of the thalamus in cognitive processing has been well documented, especially involving memory deficits [1], but literature regarding cognitive defects in thalamic lesions is sadly lacking. There are a handful of papers that describe the effects of thalamic lesions on cognitive function. One such paper by Liebermann et al. [2] compared Wisconsin Card Sorting Test (WCST) (measures 'set shifting', which is an indicator of executive function) scores in 19 patients with focal thalamic lesions with 20 healthy volunteers. In eight patients with thalamic lesions, a deficit in WCST score was recognized. The involvement of the thalamus in memory is well appreciated. A recent review by Carlesimo [3] and colleagues aimed to improve understanding about memory deficits in patients with thalamic lesions. Lacunar infarcts that result in mesial and anterior thalamic lesions are known to cause amnesic syndromes in humans. Carlesimo and colleagues [3] found 41 papers yielding 83 patients with relevant pathology. They found that these patients had similar deficits to mesio-temporal lobe damaged amnesics. That means that they displayed deficits of declarative anterograde long-term memory and spared shortterm and implicit memory. Caballero [4] did a retrospective review of 1253 patients with ischemic stroke. He found and reported 10 cases of bilateral paramedian thalamic lesions on MRI and described the consistent clinical picture that this pathology causes. The patients had disorders of consciousness, memory problems, vertical gaze paresis and psychological changes. The cognitive changes tended to persist. In a similar study by Weidauer and co-workers [5], they found that $58 \%$ of 38 patients with paramedian thalamic infarcts had cognitive deficits.
In terms of the integration of the thalamic nuclei within corticostriatal-thalamo-cortical, Metzger et al [6] showed, by means of high resolution $\mathrm{fMRI}$, different thalamic nuclei co-activating with different structures. The mediodorsal nucleus of thalamus coactivated with the pregenual anterior cingulate cortex (pgACC) as a distinct cognitive cortico-striato-thalamo-cortical "loop".

The Stroop Color Word Interference test [7] is a well-known task designed to measure several cognitive constructs including sustained attention, interference, and inhibition [8,9]. The Stroop test consists of colour name words printed in both congruent colours (i.e. blue printed in blue ink) and incongruent colours (i.e. blue printed in red ink). Naming the colour of the ink when the meaning of the word is an incongruent colour results in a delayed response time, known as Stroop interference [9]. Such interference is due to the effortless nature of reading, and the less habitual behavior of colour naming [10,11].

*Corresponding author: Katarzyna Kucharska, Institute of Psychiatry and Neurology, 9 Sobieski 02-957 Warsaw, Poland, Tel: +48 (22) 4582 607; Fax: +48 (22) 4582 566; E-mail: kate.pietura@googlemail.com

Received September 11, 2015; Accepted October 07, 2015; Published October 14,2015

Citation: Kucharska K, Wilkos E, Stefański R, Makowicz G, Ryglewicz D, et al (2015) Psychological and Physiological Changes in Cognitive Functioning in Thalamic Lesion throughout the Rehabilitation Process. J Neurol Neurophysiol 6 : 318. doi:10.4172/2155-9562.1000318

Copyright: (c) 2015 Kucharska K, et al. This is an open-access article distributed under the terms of the Creative Commons Attribution License, which permits unrestricted use, distribution, and reproduction in any medium, provided the original author and source are credited. 
In healthy controls, the medial frontal region has been implicated in control of voluntary actions, especially during tasks requiring a choice between competing responses [12-15]. The anterior cingulate cortex (ACC), particularly, is involved in a large range of behavioral adjustments and cognitive controls, namely detecting conflicts caused by competition of responses/behaviors, more broadly in processing cognitively demanding tasks [16-18]. Imaging studies have confirmed increased activation within the PFC, particularly the ACC, which is proposed to be caused by the cognitive interference that results from simultaneous processing of two stimulus features with contrasting "stimulus-response" associations [19-25]. Greater conflict-related activity within the ACC associated with the high-adjustment (i.e., incongruent) trials $[17,26]$ supports a role of this region in conflict monitoring and its engagement in cognitive control [11,16,27]. The inferior frontal cortex has also been implicated in response conflict and inhibition as well as in selective attention processes [28]. The activation of the inferior frontal region during the incongruent conditions suggests the involvement of this area in restraining a more habitual response in favour of more effortful alternatives [11,28].

After-stroke rehabilitation focuses mainly on motor recovery and linguistic therapy (treatment of aphasias), omitting or marginalizing the importance of cognitive and social deficits, which obviously has significant influence on the quality of the life for patients and their independent living. There is a growing body of literature suggesting higher efficacy of after stroke cognitive rehabilitation compared to TAU with no such intervention added in the cognitive recovery process of service users [29-31]. Yoo et al. [31] described the therapeutic effect of computer-based cognitive rehabilitation on cognitive functioning of stroke patients. Authors divided forty-six patients into two subject groups via random assignment. The training group underwent five week therapy (30 minutes daily, 5 times weekly) including a computerized cognitive rehabilitation program using The RehaCom software. The control group received standard rehabilitation therapy including physical and occupational therapy. After therapy, the training group presented with statistically significant improvement of various cognitive deficits with no statistically significant improvement in activities of daily living. Other promising findings were described by Starovasnik et al. [32] in eleven patients after stroke who underwent a training of selective attention four times a week for three months.

The rationale of this paper is: 1) to assess psychological and physiological changes in cognitive functioning of the patient with left ischemic lesion localized in tuberothalamic territory who underwent stroke rehabilitation involving treatment as usual (TAU) combined with Neurocognitive Training (NT) with a view to the remediation of cognitive functioning, 2) to examine how the anatomical reorganization post-stroke would affect cognitive functioning.

\section{Subjects and Methods}

\section{Subjects}

A 52 year-old right-handed male was admitted to the hospital after temporal disorientation and significant word finding difficulty with secondary comprehension and verbal memory problems. The patient was in good health except for a long-standing history of hypertension and obesity.

At admission, the patient was fully awake and co-operative. Neurologic evaluation revealed anomia with decreased verbal output and impaired fluency. Impairment of comprehension and semantic paraphasic errors were also noted. In striking contrast, repetition, reading and writing were well preserved. Digit recall, five-minute recall of three objects and long term memory were impaired. As a result of his neurological impairment and cognitive deficits, B.D's daily functioning including social interactions was moderately affected. Cranial nerve, motor system, reflexes, cerebellar and sensory systems were normal. Basic biochemistry, full blood counter, liver and kidney function tests and thyroid function tests were normal.

Unenhanced cranial computerized tomogram (CT) scan revealed an area of low density in the left thalamus. Magnetic resonance imaging of brain (including sagittal and axial T1, T2, FLAIR, axial diffusion weighted images and coronal gradient echo images) showed a left hemisphere ischemic lesion in the tuberothalamic area of vascular supply (19x10 $\mathrm{mm}$ in size).

The angiotensin-convering enzyme inhibitor enalapril was introduced at the dose of $5 \mathrm{mg}$ once daily as a part of TAU, and his memory and speech disturbances gradually improved over the subsequent 9 days.

The patient was not treated psychiatrically and there were no mood or anxiety problems on initial assessment whilst he scored low on the following scales: The State-Trait Anxiety Inventory (STAI) by Spielberger, STAI (state) 31, STAI (trait) 37, and Hamilton Depression Scale (HDS) -3 points.

13 healthy volunteers ( 8 male, 5 female) participated after giving informed consent. Approval for the study was obtained from the ethics committee of the Institute of Psychiatry and Neurology in Warsaw, Poland. All participants were right-handed. The cognitive state of subjects was assessed by means of the Mini-Mental State Examination (MMSE) [33]. An inclusion criterion for all subjects was a minimum score of 23. None had a history of learning disability. Exclusion criteria for all subjects included: habitual drug or alcohol abuse, secondary neurological disorders (e.g. epilepsy, dementia) and other psychiatric diagnoses. Subjects who had difficulties with vision or hearing were also excluded.

\section{Methods}

For research purposes, the researchers used standardized neuropsychological measurements that were delivered via computerized software (Cogstate) [34].

Various neurocognitive functions were measured using well known tests/batteries:

a) Benton Visual Retention Test (BVRT), method A - visuospatial processing, visual memory;

b) California Verbal Learning Test (CVLT) - learning and remembering verbal material;

c) The Rey-Osterrieth Complex Figure Test (ROCF) - visuospatial abilities, executive functions;

d) Trail Making Test (TMT, part A and B) - test of visual attention and attentional switching;

e) Color - Word Stroop Task (CWST) - attention, executive functioning;

f) Verbal Fluency Tests (VFT) - semantic memory, executive functioning;

g) Cogstate - Groton Maze Learning Test (GML), Groton Maze 
Learning Test-Delayed Recall (GML-DR), Detection Task (DT), Identification Task (IT), One Card Learning Task (OCLT), One Back Task (OBT), Two Back Task (TBT), Set-Shifting Task (S-ST) - attention, learning and memory, and executive functioning/spatial problem solving [34].

In addition, the following clinical scales were used: the MMSE, The STAI, and HDS.

\section{Neurocognitive Training (NT)}

The computer-based NT used in this study is one of the cognitive rehabilitation programs incorporating elements of Cognitive Remediation Therapy (CRT), Problem-Solving Therapy (PST), and Meta-Cognitive Training (MCT) [35]. The Neurocognitive Training equips people with a comprehensive stimulation of cognitive functions through various modules of Cogpack software (version 8.6) allowing a service user to adjust the degree of difficulty to his current cognitive level. NT was delivered by a trained psychologist (E.W.) once weekly for 60 minutes in a 12-week program (12 sessions) delivered on ward or after discharge in out-patient clinic setting. NT is a method based on metacognitive work focusing on deficits in processing information and improving cognitive strategies. Providing cognitive tasks gives the participants a chance to see their own strong and weak points within attention (selective, divided, alternating attention), memory (especially short-term memory), visual and spatial functions, abstract and holistic thinking and executive functions such as planning, cognitive flexibility, problem solving.

NT is based on so called ecological validity, which at its core places systemic approach to both an individual and to their environment, as well as to interdependences between them. The training is supplemented with mindfulness exercises, assisting the process of adaptation to changes brought about by the illness and aiding dealing with stress which accompanies it. These types of exercises influence the improvement of focusing attention and creating conditions conducive to acquisition of new skills.

\section{Neuroimaging methods}

Structural and functional magnetic resonance imaging (fMRI) investigations in the control and the experimental group were performed on a $1.5 \mathrm{~T}$ clinical imaging system (Philips Achieva Nova Dual; wholebody system, Philips Medical Systems, Warsaw, Poland). The study utilized primary fluid-attenuated inversion recovery, diffusion weighted imaging, and T1 sequences for diagnosis of thalamic lesions and the exclusion of other pathologies of the brain. The scans were read by a radiology specialist with eight years of experience (G.M.). The criteria that were used to determine the presence of unilateral lesions were hyperintense changes in diffusion weighted images and a corresponding decrease in the apparent diffusion coefficient maps, usually with visible outbreak in fluid-attenuated inversion recovery in this area.

\section{Image acquisition}

Neuroimaging data were collected using a 1.5T Philips MRI scanner at the Institute of Psychiatry and Neurology. Structural 3D Sagittal MP-RAGE images were acquired with the following parameters: TE: $4 \mathrm{~ms}$, TR: $8.67 \mathrm{~ms}$, voxel $1 \mathrm{mmx} 1 \mathrm{mmx} 1 \mathrm{~mm}$, image matrix: $240 \times 240$, 170 slices. Blood-oxygenation-level-dependent (BOLD) images were acquired with a reverse gradient-echo EPI sequence: 30 axial slices (4.5 $\mathrm{mm}$ thick, $0 \mathrm{~mm}$ gap; TR $/ \mathrm{TE}=3000 / 50 \mathrm{msec}, \mathrm{FOV}=220 \mathrm{~mm}$, matrix $=92$ $\mathrm{x}$ 92), parallel to the AC-PC line and encompassed the entire cerebrum and the majority of the cerebellum.

\section{Stroop task}

Stimuli consisted of words printed in one of four colours (red, blue, green, or yellow) to form two conditions. The Congruent condition (C) consisted of colour words printed in the same colour as the semantic meaning of the word (e.g. "blue" in blue ink). The Incongruent condition (I) consisted of colour words printed in a colour different from the meaning of the word (e.g. "red" printed in blue ink). Stimuli were presented centrally. Each stimulus was presented on a black background (duration 100ms), preceded by fixation cross $(1500 \mathrm{~ms}$ ' + '), and followed by a black screen $(2100 \mathrm{~ms})$ - decision. Participants responded using a 2-button MRI-compatible fiber optic response pad (NNL) Right hand decision - thumb-congruent, index fingerincongruent. Stimuli were presented and responses were recorded using NNL. The task was presented to participants on a screen located at the foot of the patient table, which participants viewed through a mirror mounted on the head coil, and all lighting in the scanning room was turned off.

Participants were instructed to respond as quickly and accurately as possible and to do their best. Prior to imaging, participants were also required to view the task outside the scanner and performed one block of each condition to ensure accurate performance. Each trial was presented in blocks of 10 trials in the sequence congruent-incongruent 10 times (200 trials, 248 volumes).

\section{Data preprocessing and analysis}

Data preprocessing was performed using SPM8 (http://www.fil. ion.ucl.ac.uk/spm). Functional data for each participant were realigned using the first slice as a reference, and unwrapped to correct for static inhomogeneity of the magnetic field and movement by inhomogeneity interactions. Functional volume was co-registered to the subject's MPRAGE image. The subject's MPRAGE image was segmented. Data were normalized to Montreal Neurological Institute standardized template and spatially smoothed with a Gaussian kernel of 8-mm fullwidth at half-maximum.

\section{Results}

\section{Neuropsychological results}

B.D underwent neuropsychological examination twice: three weeks after stroke onset, and after 12 weeks of participation in NT training.

In Cogstate significant changes were noted between the first and the second measurement in the GML, involving working memory and spatial problem solving. In the first measurement the patient made 60 errors, while in the second study reported 34 mistakes. We could conclude that there were improved learning processes and visual shortterm memory. A significant improvement was noted also in GMR, which is a repetition of the GML test, taken after approx. 30 minutes. In the first study, the patient made 11 errors and in the second three errors, which indicated a very good result in terms of the reminder after deferring time (visual long-term memory).

The most common mistakes observed in the GML and GMR during the first examination were return to the front, breaking the rules and perseverations. In the second study, the patient made significantly fewer errors in the breaking of rules and perseveration. We observed also extending time for the performance of tasks, especially in the test GMR.

In the remaining six tests included in the battery, there were no significant differences in execution before and after NT. 
Citation: Kucharska K, Wilkos E, Stefański R, Makowicz G, Ryglewicz D, et al. (2015) Psychological and Physiological Changes in Cognitive Functioning in Thalamic Lesion throughout the Rehabilitation Process. J Neurol Neurophysiol 6: 318. doi:10.4172/2155-9562.1000318

Parameters evaluated in CVLT also changed for the better. This allowed us to come to the conclusion that there was improvement in memory processes, learning, active reproduction and recognizing verbal material, reproduction with the instructions, and categorical learning. The overall result in the Immediate Recall remained within normal limits in both studies (5 sten), while improvement ensued in the indicator Short Delay Free Recall (in sequence 3 and 8 sten) and in Long Delay Free Recall (respectively 5 and 8 sten).

The improvement may indicate increased ability to efficiently maintain words, both in the short and long term memory, and also in recall of the learned verbal material and involved the use by the patient of the memory strategies which he learned during NT. The patient managed somewhat worse than in the first study in Long Delay Recognition; although the result of correct recognitions was still within normal range (respectively 6 and 5 sten), he made 1 error.

There were improved results in The Color - Word Stroop Task (task interference), whose completion took B.D. 82 seconds compared with 105 seconds in the first study, wherein he made one error in both measurements. Both the first and the second result were abnormal and may indicate an impaired ability to switch attention and executive control equated with resistance to interference. There were also changes in the results in TMT, which was used to assess attention and visuospatial processes, the rate of mental work as well as executive functions, such as the ability to control the course of actions. Before NT performance of part A took B.D. 29 seconds (75 percentile), and part B, 70 seconds (75-90 percentile). After the NT, part B was completed in 58 seconds (closer to the 90 percentile). These results in both studies remained within normal range.

The patient achieved mild improvement in verbal fluency tests, both semantic and phonetic. He had difficulty with generating words that begin with a definite sound.

B.D. showed difficulty in the task of repeating digits backwards. He was able to repeat only three digits, which might have indicated a reduction in his working memory and attentional switching on the numeral material. He coped better with repeated directly digits, with improvement of four in the first test and five in the second, which indicated the proper scope of the direct memory and attention (Table 1).

\section{Behavioral data for the Stroop task}

For healthy participants, reaction times (RT) for two conditions of the Stroop task were analyzed. In the congruent condition averaged RT were shorter (mean 732 ms, standard error of measurement SE 122 $\mathrm{ms}$ ) than in the incongruent condition (mean $838 \mathrm{~ms}$, SE $146 \mathrm{~ms}$ ). We also checked the number of correct and incorrect classification, and for incongruent we received $87 \%$ correct responses (SE 6\%) and for congruent $89 \%$ (SE 15\%).

Reaction time for patient B.D. was shorter for congruent condition (mean $741 \mathrm{~ms}$, SE $176 \mathrm{~ms}$ ) than for incongruent (mean $782 \mathrm{~ms}$, SE 278 $\mathrm{ms}$ ) and were shortened after therapy for both conditions (congruent condition - mean $638 \mathrm{~ms}$, SE $111 \mathrm{~ms}$, incongruent condition - mean $720 \mathrm{~ms}$, SE $178 \mathrm{~ms})$. For incongruent condition we recorded a small change in correct responses, from $96 \%$ before to $98 \%$ after therapy.

\section{fMRI results}

The results were thresholded at $\mathrm{z}=2.3$ and corrected for multiple comparisons at cluster significance level of $\mathrm{P}<0.05$.

\section{C-I comparison}

B.D

In response to the Stroop task in acute stroke before rehabilitation program, significant activation clusters for the C-I comparison are reported in Table 2 .

By contrasting the congruent vs the incongruent condition, after treatment activations only in the left parietal lobule and right middle frontal gyrus remained (Figure 1).

\section{Healthy controls}

Significant activation clusters for the C-I comparison are reported in Table 3 and Figure 2.

\section{I-C comparison}

\section{B.D}

Before therapy significant activation clusters for the I-C comparison are reported in Table 2 .

By contrasting the incongruent versus congruent condition,

\begin{tabular}{|c|c|c|c|c|}
\hline Variable & Measure & $H C(n=13)$ & B.D. Before Therapy & B.D. After Therapy \\
\hline Age & Years $(X \pm S D)$ & $50.92 \pm 12.42$ & 52 & 52 \\
\hline Education & Years $(X \pm S D)$ & $17.08 \pm 2.87$ & 17 & 17 \\
\hline MMSE & 30 scorable responses & $29.09 \pm .94$ & 26 & 29 \\
\hline Hamilton & 21 scorable responses & $7.46 \pm 4.68$ & 3 & 5 \\
\hline STAI anxiety state & $(X \pm S D)$ & $38.45 \pm 10.59$ & 31 & 34 \\
\hline STAI anxiety trait & $(X \pm S D)$ & $43.45 \pm 9.56$ & 37 & 37 \\
\hline Stroop Test & time in sec & $59.90 \pm 20.65$ & 105 & 82 \\
\hline Stroop Test & errors numer & $0.81 \pm 1.40$ & 1 & 1 \\
\hline GML & total errors & $51.58 \pm 10.77$ & 60 & 34 \\
\hline GML & duration (ms) & $242021.58 \pm 575.38$ & 267199 & 294739 \\
\hline GMR & total errors & $8.41 \pm 2.06$ & 11 & 3 \\
\hline GMR & duration (ms) & $40048.50 \pm 9066.06$ & 28064 & 41410 \\
\hline TMT A & duration (sec) & $31.90 \pm 8.83$ & 29 & 28 \\
\hline TMT B & duration (sec) & $69.80 \pm 15.62$ & 70 & 58 \\
\hline CVLT Immediate Recall & time in seconds & $57.18 \pm 9.57$ & 44 & 50 \\
\hline
\end{tabular}

MMSE - Mini-Mental State Examination; STAI - The State-Trait Anxiety Inventory ; GML - Groton Maze Learning Test; GMR - Groton Maze Recall; TMT - Trail Making Test; CVLT - California Verbal Learning Test; X- mean value, SD - standard Deviation

Table 1: Demographic and clinical data for the healthy controls (HC) and the patient (B.D) before and after 12 week therapy 
Citation: Kucharska K, Wilkos E, Stefański R, Makowicz G, Ryglewicz D, et al. (2015) Psychological and Physiological Changes in Cognitive Functioning in Thalamic Lesion throughout the Rehabilitation Process. J Neurol Neurophysiol 6: 318. doi:10.4172/2155-9562.1000318

Page 5 of 7

B.D: Before Therapy

\section{Congruent-Incongruent Comparison}

\begin{tabular}{|l|c|c|c|c|}
\hline Location & Hemisphere & X & Y & Z \\
\hline Superior Frontal Gyrus & L & -15 & 60 & 18 \\
\hline Medial frontal Gyrus & L & -12 & 45 & 18 \\
\hline Middle Frontal Gyrus & $\mathrm{R}$ & 48 & 39 & 27 \\
\hline Frontal Inf Orb & $\mathrm{L}$ & -39 & 51 & -15 \\
\hline Fusiform & $\mathrm{R}$ & 27 & 0 & -39 \\
\hline Cerebellum & $\mathrm{L}$ & -45 & -75 & -39 \\
\hline Cerebellum & $\mathrm{R}$ & 18 & -84 & -36 \\
\hline Incongruent-Congruent Comparison & & & & \\
\hline Location & Hemisphere & $\mathrm{X}$ & $\mathrm{Y}$ & $\mathrm{Z}$ \\
\hline Precentral Gyrus & $\mathrm{L}$ & -33 & -10 & 72 \\
\hline Postcentral Gyrus & $\mathrm{L}$ & -40 & -39 & 72 \\
\hline Middle Frontal Gyrus & $\mathrm{L}$ & -27 & 44 & 48 \\
\hline Precentral Gyrus & $\mathrm{R}$ & 44 & -16 & 68 \\
\hline Insula & $\mathrm{L}$ & -34 & 15 & 10 \\
\hline
\end{tabular}

B.D: After Therapy

Congruent -Incongruent Comparison

\begin{tabular}{|l|c|c|c|c|}
\hline Location & Hemisphere & X & Y & Z \\
\hline Inferior Parietal Lobule & L & -51 & -51 & 54 \\
\hline Frontal Middle Gyrus & R & 39 & 27 & 54 \\
\hline
\end{tabular}

Incongruent - Congruent Comparison

\begin{tabular}{|l|c|c|c|c|}
\hline Location & Hemisphere & X & Y & Z \\
\hline Lateral Occipital Cortex, Precuneous & R & 10 & -66 & 68 \\
\hline Cerebellum & R & 28 & -50 & -57 \\
\hline Inferior Frontal Gyrus & L & -49 & 25 & -8 \\
\hline Frontal Lobe, Precentral Gyrus & L & -46 & 8 & 38 \\
\hline Middle Temporal Gyrus & L & -56 & -12 & -12 \\
\hline $\begin{array}{l}\text { Superior Parietal Lobule, } \\
\text { Lateral Occipital Cortex, }\end{array}$ & L & -12 & -66 & 64 \\
\hline
\end{tabular}

Table 2: Brain activation in response to Stroop task in B.D before and after rehabilitation program. after treatment significant activations were reported in the following brain regions: right lateral occipital cortex, right precuneous, right cerebellum, left middle temporal gyrus, and frontal regions (left inferior frontal gyrus, left frontal lobe, precentral gyrus) (Figure 1).

\section{Healthy controls}

Significant activation clusters for the I-C comparison are reported in Table 3 and Figure 2.

\section{Discussion}

This study examined the neural correlates of efficient rehabilitation program (TAU combined with NT) in a patient with left tuberothalamic lesion.

To the authors' knowledge this is the first study to show efficacy of the above mentioned rehabilitation program in a case of unilatera thalamic stroke as compared to the healthy control group.

The clinical syndrome resulted from infarction in the territory of the tuberothalamic artery manifested in the form of various

\begin{tabular}{|l|c|c|c|c|}
\hline Congruent - Incongruent Comparison \\
\hline Location & Hemisphere & X & Y & Z \\
\hline Superior Frontal Gyrus (BA 6) & L & -10 & 36 & 56 \\
\hline Medial Frontal Gyrus (BA 9) & R & 6 & 50 & 20 \\
\hline Precuneus (BA 19) & R & 40 & -76 & 48 \\
\hline Supramarginal Gyrus (BA 40) & L & -64 & -46 & 26 \\
\hline Incongruent - Congruent Comparison & & & \\
\hline Location Hemisphere & & X & Y & Z \\
\hline Occipital Lobe, Cuneus & L & -10 & -98 & 18 \\
\hline Posterior Cingulate (BA 30) & R & 26 & -66 & 10 \\
\hline Sublobar, Insula & R & 46 & -4 & 14 \\
\hline Superior Frontal Gyrus (BA 6) & R & 4 & 10 & 52 \\
\hline
\end{tabular}

Table 3: Brain activation in response to Stroop task in the healthy controls. The results were thresholded at $z=2.3$ and corrected for multiple comparisons at cluster Significance level of $\mathrm{P}<0.05$
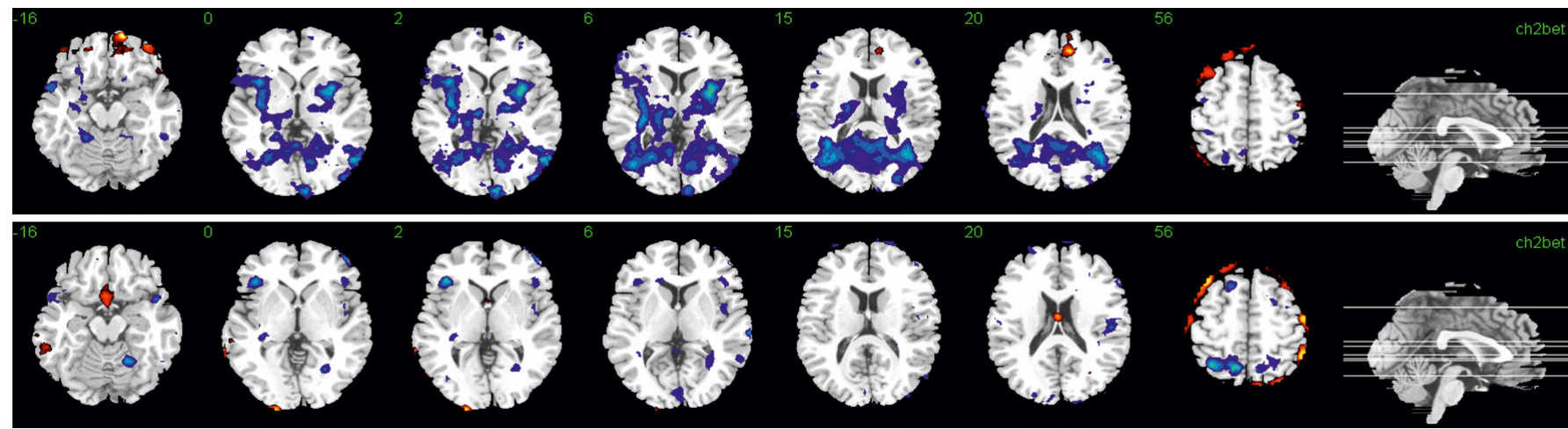

Figure 1: Brain map of neural activation in response to Stroop task in acute stroke B.D. before (A) and after rehabilitation program (B). Clusters were identified using False Discovery Rate (FDR) correction of $p<0.05$. Red - results for comparison congruent vs incongruent. Blue - results for comparison incongruent vs congruent.

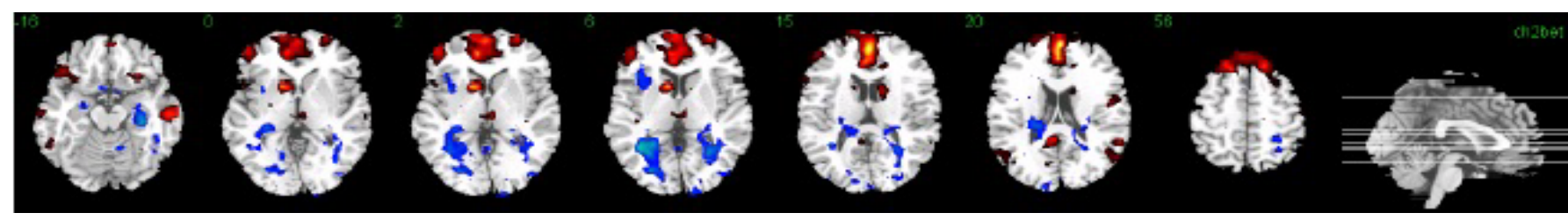

Figure 2: Brain maps of neural activation in response to Stroop task in the healthy control Group Red (C vs I) and blue (I vs C). 
Citation: Kucharska K, Wilkos E, Stefański R, Makowicz G, Ryglewicz D, et al. (2015) Psychological and Physiological Changes in Cognitive Functioning in Thalamic Lesion throughout the Rehabilitation Process. J Neurol Neurophysiol 6: 318. doi:10.4172/2155-9562.1000318

neuropsychological deficits, mostly of a cognitive nature [36]. The most prominent features of left tuberothalamic stroke are impairments in recent memory and in new learning. Language disturbances usually occur in left hemisphere lesions in the form of impaired fluency and comprehension [36].

In our study, the stroke in B.D. was confined to the most anterior region of the thalamus and his presentation was considerably different from those with the larger tuberothalamic infarcts. The patient's ability to comprehend, write, and read remained intact; however, there were deficits in new learning, memory and attention.

Memory deficits in tuberothalamic infarcts may represent a disconnection involving two pathways: 1) between anterior thalamic nuclei and hippocampal formation in virtue of the disruption of the mammillothalamic track, and 2) between amygdala and anterior nuclei by damage to the amygdalothalamic projections passing through the internal medullary lamina [37].

Concurrently with clinical improvement, marked post-therapy changes were identified in cognitive functioning. With regard to neuropsychological assessment, B.D. presented with clinically significant post-therapy improvement in his neurocognitive functions containing memory, learning, and attentional switching.

The patient's initial response to the Stroop task before therapy (congruent versus incongruent) recruited the frontal regions (left superior, bilateral middle, and left inferior orbital), right fusiform gyrus and bilateral cerebellum whereas by contrasting the incongruent versus congruent condition, statistically significant activations were reported in bilateral precentral gyri, left postcentral gyrus, left middle frontal gyrus, and left insula.

B.D. showed a post-therapy contraction of activation pattern and by contrasting the congruent versus the incongruent condition, after treatment only left parietal lobule and right middle frontal gyrus remained more significantly activated in response to the Stroop task. By contrasting the incongruent versus congruent condition, after treatment increased BOLD signal was reported in right lateral occipital cortex, right precuneous, right cerebellum, left middle temporal gyrus, and frontal regions (left inferior frontal gyrus, left frontal lobe, precentral gyrus).

Our fMRI results in the healthy controls showed activation in right anterior cingulate, frontal regions (middle, superior, and orbital part of the inferior frontal gyri) and striatal region during Stroop interference, which remains in line with other studies [9,38-40]. The ACC is thought to monitor conflict or error during response selection $[26,39,41,42]$, while inhibitory control during response execution primarily involved the VLPFC $[39,43]$ and the striatum $[39,44-46]$.

In the Stroop task, the target and the distractor are different attributes of the same object. In addition, due to the automatic nature of reading the distracting material, an incorrect response is highly reported in incongruent trials. Therefore, in the Stroop task, there appears to be a greater demand for selective attention to filter out the distracting information. In the event that selective attention fails to filter out irrelevant information completely, it is likely that the irrelevant information will bias toward the inappropriate response [37].

Summing up, the thalamus plays a considerable role in cognitive processing.

Further research is needed to increase understanding about efficacy of cognitive remediation and the theory of neuroplasticity of potential anatomical reorganization post-stroke and its impact on cognitive functioning.

\section{Acknowledgment}

This research project is supported by National Centre of Science (NCN) grant (Poland) 2011/01/B/NZ5/02838.

Many thanks to Dr. Natasza Nalesnik from the Institute of Psychiatry in London for useful comments on the manuscript and Blazej Sawionek and Piotr Bogorodzki for their help with scanning procedure.

\section{References}

1. Metzger CD, van der Werf YD, Walter $M(2013)$ Functional mapping of thalamic nuclei and their integration into cortico-striatal-thalamo-cortical loops via ultrahigh resolution imaging-from animal anatomy to in vivo imaging in humans Front. Neurosci 7:24.

2. Liebermann D, Ploner CJ, Kraft A, Kopp UA, Ostendorf F (2013) A dysexecutive syndrome of the medial thalamus. Cortex 49: 40-49.

3. Carlesimo GA, Lombardi MG, Caltagirone C (2011) Vascular thalamic amnesia a reappraisal. Neuropsychologia 49: 777-789.

4. Jiménez Caballero PE (2010) Bilateral paramedian thalamic artery infarcts: report of 10 cases. J Stroke Cerebrovasc Dis 19: 283-289.

5. Weidauer S, Nichtweiss M, Zanella FE, Lanfermann H (2004) Assessment of paramedian thalamic infarcts: MR imaging, clinical features and prognosis. Eur Radiol 14: 1615-1626.

6. Metzger CD (2010) High field fMRI reveals thalamocortical integration of segregated cognitive and emotional processing in mediodorsal and intralaminar thalamic nuclei, Front. Neuroanat 4: 138.

7. Stroop JR (1935) Studies of Interference in Serial Verbal Reactions, J. Exp. Psychol 18: 643-662.

8. Barkley RA (1997) Behavioral inhibition, sustained attention, and executive functions: constructing a unifying theory of ADHD. Psychol Bull 121: 65-94.

9. Langenecker SA, Nielson KA, Rao SM (2004) fMRI of healthy older adults during Stroop interference. Neuroimage 21: 192-200.

10. MacLeod CM (1991) Half a century of research on the Stroop effect: an integrative review. Psychol Bull 109: 163-203.

11. Ovaysikia S, Tahir KA, Chan JL, DeSouza JF (2011) Word wins over face: emotional Stroop effect activates the frontal cortical network. Front Hum Neurosci 4: 234

12. Schlag-Rey M, Amador N, Sanchez H, Schlag J (1997) Antisaccade performance predicted by neuronal activity in the supplementary eye field Nature 390: 398-401

13. Carter CS, Botvinick MM, Cohen JD (1999) The contribution of the anterior cingulate cortex to executive processes in cognition. Rev Neurosci 10: 49-57.

14. Ito S, Stuphorn V, Brown JW, Schall JD (2003) Performance monitoring by the anterior cingulate cortex during saccade countermanding. Science 302: 120122.

15. Sumner $P$, Nachev $P$, Morris $P$, Peters AM, Jackson SR et al. (2007) Human medial frontal cortex mediates unconscious inhibition of voluntary action, Neuron 54: 697-711.

16. Carter CS, Braver TS, Barch DM, Botvinick MM, Noll D, et al. (1998) Anterior cingulate cortex, error detection, and the online monitoring of performance. Science 280: 747-749.

17. Kerns JG, Cohen JD, MacDonald AW 3rd, Cho RY, Stenger VA, et al. (2004) Anterior cingulate conflict monitoring and adjustments in control. Science 303 : 1023-1026.

18. Fellows LK, Farah MJ (2005) Is anterior cingulate cortex necessary for cognitive control? Brain 128: 788-796.

19. Pardo JV, Pardo PJ, Janer KW, Raichle ME (1990) The anterior cingulate cortex mediates processing selection in the Stroop attentional conflict paradigm. Proc Natl Acad Sci U S A 87: 256-259.

20. Bench CJ, Frith CD, Grasby PM, Friston KJ, Paulesu E, et al. (1993) Investigations of the functional anatomy of attention using the Stroop test. Neuropsychologia 31: 907-922. 
Citation: Kucharska K, Wilkos E, Stefański R, Makowicz G, Ryglewicz D, et al. (2015) Psychological and Physiological Changes in Cognitive Functioning in Thalamic Lesion throughout the Rehabilitation Process. J Neurol Neurophysiol 6: 318. doi:10.4172/2155-9562.1000318

Page 7 of 7

21. Vendrell P, Junqué C, Pujol J, Jurado MA, Molet J, et al. (1995) The role of prefrontal regions in the Stroop task. Neuropsychologia 33: 341-352.

22. Bush G, Whalen PJ, Rosen BR, Jenike MA, Mclnerney SC, et al. (1998) The counting Stroop: an interference task specialized for functional neuroimaging-validation study with functional MRI. Hum Brain Mapp 6: 270-282.

23. Barch DM, Braver TS, Akbudak E, Conturo T, Ollinger J, et al. (2001) Anterior cingulate cortex and response conflict: effects of response modality and processing domain. Cereb Cortex 11: 837-848.

24. Zysset S, Müller K, Lohmann G, von Cramon DY (2001) Color-word matching stroop task: separating interference and response conflict. Neuroimage 13: 2936 .

25. Melcher T, Gruber O (2009) Decomposing interference during Stroop performance into different conflict factors: an event-related fMRI study. Cortex 45: $189-200$.

26. MacDonald AW 3rd, Cohen JD, Stenger VA, Carter CS (2000) Dissociating the role of the dorsolateral prefrontal and anterior cingulate cortex in cognitive control. Science 288: 1835-1838.

27. Botvinick MM, Cohen JD, Carter CS (2004) Conflict monitoring and anterior cingulate cortex: an update. Trends Cogn Sci 8: 539-546.

28. Kemmotsu N, Villalobos ME, Gaffrey MS, Courchesne E, Müller RA (2005) Activity and functional connectivity of inferior frontal cortex associated with response conflict. Brain Res Cogn Brain Res 24: 335-342.

29. Cha YJ, Kim H (2013) Effect of computer-based cognitive rehabilitation (CBCR) for people with stroke: a systematic review and meta-analysis. NeuroRehabilitation 32: 359-368.

30. Park JH (2015) The effects of a Korean computer-based cognitive rehabilitation program on cognitive function and visual perception ability of patients with acute stroke," J. Phys. Ther. Sci 27: 2577-2579.

31. Yoo C, Yong MH, Chung J, Yang Y (2015) Effect of computerized cognitive rehabilitation program on cognitive function and activities of living in stroke patients. J Phys Ther Sci 27: 2487-2489.

32. Starovasnik Agavec B, Mlinaria Leajnik V, Goljar N (2015) Training of selective attention in work-active stroke patients. Int J Rehabil Res .

33. Folstein MF, Folstein SE, McHugh PR (1975) 'Mini-mental state'. A practical method for grading the cognitive state of patients for the clinician, J. Psychiatr. Res 12: 189-198.
34. Wilkos E, Brown TJ, Slawinska K, Kucharska KA (2015) Social cognitive and neurocognitive deficits in inpatients with unilateral thalamic lesions - pilot study. Neuropsychiatr Dis Treat 11: 1031-1038.

35. Kucharska-Pietura K, Wilkos E, Sawicka M (2013) Trening spolecznego poznania i neuropoznania: podrecznik terapeuty. Warszawa: Instytut Psychiatrii i Neurologii.

36. Schmahmann JD (2003) Vascular syndromes of the thalamus. Stroke 34: 2264 2278.

37. Graff-Radford NR, Tranel D, Van Hoesen GW, Brandt JP (1990) Diencephalic amnesia. Brain $113: 1-25$.

38. Banich MT, Milham MP, Atchley R, Cohen NJ, Webb A et al. (2000) fMRI studies of Stroop tasks reveal unique roles of anterior and posterior brain systems in attentional selection, J. Cogn. Neurosci 12: 988-1000.

39. Nee DE, Wager TD, Jonides J (2007) Interference resolution: insights from a meta-analysis of neuroimaging tasks. Cogn Affect Behav Neurosci 7: 1-17.

40. Laird AR, Fox PM, Price CJ, Glahn DC, Uecker AM et al. (2005) ALE metaanalysis: controlling the false discovery rate and performing statistical contrasts Hum. Brain Mapp 25: 155-164.

41. Pochon JB, Riis J, Sanfey AG, Nystrom LE, Cohen JD (2008) Functional imaging of decision conflict. J Neurosci 28: 3468-3473.

42. Liu X, Banich MT, Jacobson BL, Tanabe JL (2006) Functional dissociation of attentional selection within PFC: response and non-response related aspects of attentional selection as ascertained by fMRI, Cereb. Cortex 16: 827-834.

43. Rubia K, Russell T, Bullmore ET, Soni W, Brammer MJ et al. (2001) An fMR study of reduced left prefrontal activation in schizophrenia during normal inhibitory function, Schizophr. Res 52: 47-55.

44. Ali N, Green DW, Kherif F, Devlin JT, Price CJ (2010) The role of the left head of caudate in suppressing irrelevant words. J Cogn Neurosci 22: 2369-2386.

45. Li CS, Huang C, Yan P, Bhagwagar Z, Milivojevic V, et al. (2008) Neura correlates of impulse control during stop signal inhibition in cocaine-dependent men. Neuropsychopharmacology 33: 1798-1806.

46. Pompei F, Jogia J, Tatarelli R, Girardi P, Rubia K, et al. (2011) Familial and disease specific abnormalities in the neural correlates of the Stroop Task in Bipolar Disorder. Neuroimage 56: 1677-1684. 